\title{
Complex Topology of the Magnetic Field in Strong Flares
}

\author{
R.N. Ikhsanov ${ }^{1}$, Yu.V. Marushin ${ }^{1}$ and N.R. Ikhsanov ${ }^{1,2}$ \\ ${ }^{1}$ Pulkovo Observatory, St. Petersburg, Russia email: solar@gao.spb.ru \\ ${ }^{2}$ Korea Astronomy Observatory, Taejon, Republic of Korea email: ikhsanov@kao.re.kr
}

We report the " $5+1$ " dynamical classification of the most frequently observed topologies of the magnetic field in sunspot groups associated with powerful flares (see Fig.1). The classification is based on the analysis of magnetographic and $\mathrm{H} \alpha$ observations of more than 600 active regions on the span of 23 years (for detailed description see Ikhsanov 1982, Ikhsanov 1985).

Statistical analysis of the processed data revealed 3 basic types of the magnetic field topology (dynamical classes I-III) in the sunspot groups with $\delta$-configuration associated with powerful (proton) flares (see Table 2 in Ikhsanov \& Marushin 2003 and references therein). Our results confirm that the presence of $\delta$-configuration is a necessary but not a sufficient condition for a strong flare in an active region to occur. We find that all powerful flaring events (e.g. proton flares) investigated within our project were related to the emergence and development of the new magnetic flux tube(s) (or complexes of tubes) in an active region, which strongly interact with the magnetic complex earlier appeared in the region. It has been also recognized (see e.g. Ikhsanov 1982, Ikhsanov 1985, and Ikhsanov \& Peregud 1988) that the appearance of a flare depends on the parameters of interacting flux tubes (i.e. the spatial scales and the strength of the magnetic field) as well as on their mutual position (e.g. the magnetic field topology). There is a strong hint for a correlation between the flare parameters and the class of the field topology realized in the active region to which the flare is associated (Ikhsanov \& Peregud 1988). In particular, we find that the rate of distortions of the interacting flux ropes is directly related to the rate of energy release observed in corresponding flares.

Identification of the magnetic topology class in an active region provide us with information about possible location and spatial orientation of current sheet in the flare associated with this region. Application of this finding to the reconstruction of the flaring scenario observed in the active region HR 17901+17906 (Ikhsanov \& Marushin 1998) allows us to understand basically the observed anti-correlation between the optical $(\mathrm{H} \alpha)$ and X-ray fluxes detected during two powerful flares, which consequently appeared in this region. According to our data, the value of the ratio (X-ray flux/optical flux) is larger in the case of radially oriented current sheet, which corresponds to the classes II and III of the magnetic topology within our dynamical classification (see Ikhsanov \& Marushin 2003 and references therein).

\section{Acknowledgements}

Nazar Ikhsanov acknowledges the support of Korea Astronomy Observatory under their basic research programme. The work was partly supported by the Russian Foundation of Basic Research under the grant 03-02-17223a and the State Scientific and Technical Program "Astronomy". 


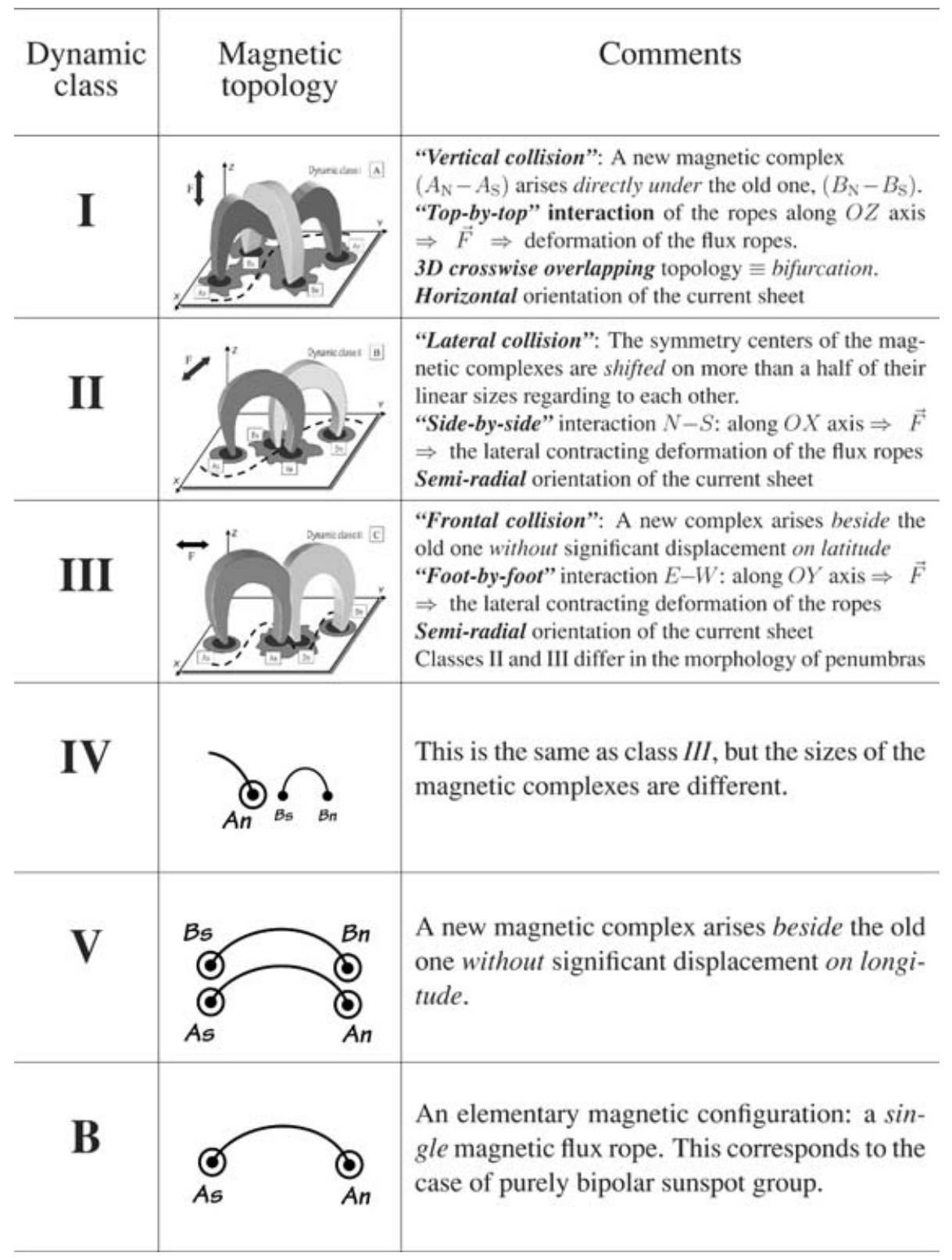

Figure 1. " $5+1 "$ Dynamic classification of the field topology

\section{References}

Ikhsanov, R.N. 1982, Izvestia GAO (Contributions of the Central Astronomical Observatory at Pulkovo), 200, 15 (in Russian)

Ikhsanov, R.N. 1985, Izvestia GAO, 201, 84 (in Russian)

Ikhsanov, R.N., Marushin, Yu.V. 1998, Izvestia GAO, 212, 91 (in Russian)

Ikhsanov, R.N., Marushin, Yu.V. 2003, astro-ph/0311114

Ikhsanov, R.N., Peregud, N.L. 1988, Solnechnye Dannye, 2, 67 (in Russian) 